\title{
Unilateral Stroke: Computer-based Assessment Uncovers Non-Lateralized and Contralesional Visuoattentive Deficits
}

\author{
Sanna Villarreal ${ }^{1 * *}$ (이 , Matti Linnavuo ${ }^{2}$, Raimo Sepponen ${ }^{2}$, Outi Vuori ${ }^{1}$, Mario Bonato ${ }^{3}$ (1) , \\ Hanna Jokinen ${ }^{1,4}$ and Marja Hietanen ${ }^{1}$ \\ ${ }^{1}$ Clinical Neurosciences, Neuropsychology, University of Helsinki and Helsinki University Hospital, Helsinki, Finland \\ ${ }^{2}$ Department of Electrical Engineering and Automation, Aalto University, Espoo, Finland \\ ${ }^{3}$ Department of General Psychology, University of Padova, Padova, Italy \\ ${ }^{4}$ Department of Psychology and Logopedics, Faculty of Medicine, University of Helsinki, Helsinki, Finland
}

(Received June 30, 2020; Final Revision December 2, 2020; Accepted December 22, 2020; First Published Online February 8, 2021)

\begin{abstract}
Objective: Patients with unilateral stroke commonly show hemispatial neglect or milder contralesional visuoattentive deficits, but spatially non-lateralized visuoattentive deficits have also been reported. The aim of the present study was to compare spatially lateralized (i.e., contralesional) and non-lateralized (i.e., general) visuoattentive deficits in left and right hemisphere stroke patients. Method: Participants included 40 patients with chronic unilateral stroke in either the left hemisphere (LH group, $n=20$ ) or the right hemisphere (RH group, $n=20$ ) and 20 healthy controls. To assess the contralesional deficits, we used a traditional paper-and-pencil cancellation task (the Bells Test) and a Lateralized Targets Computer Task. To assess the non-lateralized deficits, we developed a novel large-screen $(173 \times 277 \mathrm{~cm}) \mathrm{computer}$ method, the Ball Rain task, with moving visual stimuli and fast-paced requirements for selective attention. Results: There were no contralesional visuoattentive deficits according to the cancellation task. However, in the Lateralized Targets Computer Task, RH patients missed significantly more left-sided than right-sided targets in bilateral trials. This omission distribution differed significantly from those of the controls and LH patients. In the assessment of nonlateralized attention, RH and LH patients missed significantly more Ball Rain targets than controls in both the left and right hemifields. Conclusions: Computer-based assessment sensitively reveals various aspects of visuoattentive deficits in unilateral stroke. Patients with either right or left hemisphere stroke demonstrate non-lateralized visual inattention. In right hemisphere stroke, these symptoms can be accompanied by subtle contralesional visuoattentive deficits that have remained unnoticed in cancellation task.
\end{abstract}

Keywords: Brain damage, Visual neglect, Non-spatial attention, Selective attention, Attention impairment, Reaction times

Patients with unilateral brain damage typically show contralesional deficits in attention, the extreme form of which is hemispatial neglect (Halligan, Cockburn, \& Wilson, 1991; Heilman, Valenstein, \& Watson, 2000). Interestingly, this spatially lateralized syndrome has sometimes been associated with non-lateralized (i.e., general) deficits in selective or sustained attention (Bartolomeo \& Chokron, 2002; Husain \& Rorden, 2003; Robertson, 2001; Robertson et al., 1994). Neglect in patients with right hemisphere stroke has been, for instance, correlated with a decline in performance in sustained attention tasks (Robertson et al., 1997). Correspondingly, neglect is temporarily alleviated when

*Correspondence and reprint requests to: Sanna Villarreal, Unit of Neuropsychology, Helsinki University Hospital, P.O. Box 302, FI-00029 Helsinki, Finland. E-mail: sanna.villarreal@helsinki.fi alertness increases (George, Mercer, Walker, \& Manly, 2008; Malhotra, Parton, Greenwood, \& Husain, 2006; Mukand et al., 2001; Robertson, Mattingley, Rorden, \& Driver, 1998).

Patients with neglect can also show non-lateralized deficits in selective attention (Chokron, Peyrin, \& Perez, 2019; Husain, Shapiro, Martin, \& Kennard, 1997). Chokron and co-workers (2019) assessed the selective attention of patients with chronic right hemisphere damage by using a lateralized letter-detection task. Either a single large stimulus (low-attention version) or a small stimulus surrounded by flankers (high-attention version) was to be detected. Patients with neglect showed a bilateral deficit, which became more evident in the high-attention version compared to the lowattention version. The authors concluded that increasing the demands on visual selective attention deteriorates neglect 
patients' performance in both hemifields. Along the same lines, Battelli et al. (2001) demonstrated that right hemispheredamaged patients with neglect show a bilateral deficit in perceiving motion. A motion illusion was generated by an appropriate temporal offset between the four visual dot stimuli. It was suggested that the finding is due to a nonlateralized deficit in the temporal resolution of attention to transient events.

A clinically important aspect in these findings is the fact that if non-lateralized attention deficits are present, they modulate the lateralized ones (Robertson et al., 1997; van Kessel, van Nes, Brouwer, Geurts, \& Fasotti, 2010). This interaction aggravates and prolongs neglect (Hjaltason, Tegnér, Tham, Levander, \& Ericson, 1996; Husain \& Rorden, 2003; Robertson, 2001; Samuelsson, Hjelmquist, Jensen, Ekholm, \& Blomstrand, 1998). Previous studies have reported contradictory findings on visuoattentive deficits of patients with right hemisphere damage: studies have found a) specific contralesional omissions, while ipsilesional performance has remained intact (Bonato, 2015; Bonato, Priftis, Marenzi, Umiltá, \& Zorzi, 2012); b) mild ipsilesional deficits in addition to considerable contralesional symptoms (Bonato et al., 2019; Chokron et al., 2019); or even c) prominent ipsilesional deficits compared to contralesional performance (Robertson et al., 1994; Williamson et al., 2018). There are also methodological limitations to the applicability of past studies regarding milder forms of visuoattentive deficits. Some previous studies finding non-lateralized deficits but not lateralized ones have ruled out contralesional deficits either with a paperand-pencil method (Rueckert \& Grafman, 1998), or they did not test for neglect at all (Rueckert \& Grafman, 1996; Wilkins, Shallice, \& McCarthy, 1987). Furthermore, studies linking non-lateralized deficits with lateralized symptoms have diagnosed clear neglect with paper-and-pencil methods (Chokron et al., 2019; Robertson et al., 1997; Robertson et al., 1998). Experimental designs utilizing paper-and-pencil methods only may miss milder forms of contralesional visuoattentive deficits, and their possible connection to non-lateralized inattention. Hence, growing evidence (see, e.g., Bonato, 2015; Bonato \& Deouell, 2013; Bonato et al., 2012; Buxbaum et al. 2004; Deouell, Sacher, \& Soroker, 2005; Kim et al., 2010; Ogourtsova, Silva, Archambault, \& Lamontagne, 2017; Peskine et al., 2011; Villarreal et al., 2020) regarding the inadequacy of paper-and-pencil methods in revealing mild forms of contralesional attention deficits indicates that supplemental studies with sensitive methods are needed.

The aim of the present study was to compare spatially lateralized and non-lateralized visuoattentive deficits in left and right hemisphere stroke patients. To assess the lateralized deficits, we used a traditional paper-and-pencil cancellation task, the Bells Test, and a computer-based detection task with brief stimulus duration (Bonato et al., 2012). To assess the nonlateralized deficits, we developed a new large-screen computer method, the Ball Rain task, in which we used moving visual stimuli and a setting that requires fast-paced selective attention. We examined whether the combination of these two methodological qualities would make the assessment sensitive in revealing non-lateralized inattention and whether the same groups of patients also exhibit signs of contralesional attention deficits. To our knowledge, this is one of the first-ever comparisons between two computer methods that have already proven sensitive in detecting mild visuoattentive deficits (see Bonato et al., 2012; Villarreal et al., 2020).

\section{METHOD}

\section{Participants}

The characteristics of the participants included in this study have previously been described in detail (Villarreal et al., 2020). Briefly, patients with their first-ever neuroradiologically verified stroke (ischemic, hemorrhagic, or both) were recruited during 2016-2019 from the Neurology Outpatient Clinic of Helsinki University Hospital at 106 days after hospital admission, on average. Exclusion criteria were bilateral stroke, visual field defect in neurological or neuroophthalmological evaluation, prior neurological diagnosis affecting cognition, primary impairment in hearing or sight (other than myopia or hyperopia corrected with glasses), substance abuse, severe cognitive symptoms preventing participation, and severe psychiatric disease or significant motor symptoms complicating cooperation. Participants comprised 20 patients with right hemisphere stroke (RH patient group, 9 men, mean age 53 years, $S D \pm 8$ years), 20 patients with left hemisphere stroke (LH patient group, 15 men, mean age 51 years, $S D \pm 9$ years), and 20 healthy controls ( 8 men, mean age 46 years, $S D \pm 15$ years). There were no significant differences between the participant groups in age, gender, education, or self-reported depressive symptoms. The patient groups did not differ significantly from each other in types of stroke or in number of days after stroke prior to participating in the study (days post-onset of stroke prior to study were on average 106 (SD: 45, range: $42-235$ ) in RH patients and 105 (SD: 45, range: $39-203$ ) in LH patients).

The Ethics Committee of Helsinki University Hospital approved the study protocol, and all participants gave written informed consent for participation. The data included in the study were obtained in compliance with the Helsinki Declaration.

\section{Procedure}

\section{Assessment of contralesional visuoattentive deficits}

Prior evaluation of the patient groups' contralesional omissions has been described in detail in our previous report (Villarreal et al., 2020). To summarize, we used a traditional paper-and-pencil cancellation task, the Bells Test (Gauthier, Dehaut, \& Joanette, 1989), and two new large-screen computer methods to evaluate hemispatial neglect. According to the findings, $\mathrm{RH}$ patients had subtle neglect but LH patients showed no signs of neglect. RH patients missed significantly more left-sided unilateral targets than controls in both of the 
large-screen tasks. They also missed significantly more left-sided than right-sided unilateral targets in one of the large-screen tasks. These effects did not become evident in LH patients. No significant group differences occurred in the left-sided or right-sided omissions of the Bells Test. In the present study, we re-analyzed the results of the Bells Test by utilizing an asymmetry index instead of raw omission data (see Data analyses) and we used a Lateralized Targets Computer Task (Bonato et al., 2012). As in our previous work (Villarreal et al., 2020) and in other recent findings (Blini et al., 2016; Bonato, 2015; Bonato et al., 2012), contralesional deficits were interpreted as being only subtle if the predominance of contralateral omissions became evident with the Lateralized Targets Computer Task but not with the Bells Test.

Lateralized targets computer task. Participants performed a Finnish version of a computer-based task from the test battery developed by Bonato and co-workers. The method has been described in detail in previous studies (Blini et al., 2016; Bonato, 2015; Bonato et al., 2012). The paradigm was administered using E-Prime (Psychology Software Tools, Sharpsburg, Pennsylvania, USA, http://www.pstnet.com/).

Participants sat approximately $60 \mathrm{~cm}$ from a 15 -inch computer monitor. Each trial started with an empty white screen (1000 ms) followed by a central fixation (black central cross, $1000 \mathrm{~ms}$ ). Then, three different stimuli were presented concurrently: a black dot target(s) (diameter $8 \mathrm{~mm}$, presented for $50 \mathrm{~ms}$ ), a central letter (font size 38, same duration), and a spoken number. The dot target(s) appeared with the same probability on the left, on the right, or bilaterally (one target type in each trial, altogether 16 of each target type) at a distance of $135 \mathrm{~mm}$ (about $12.8^{\circ}$ ) from the center. The central letter $(a, b, v$, or $z$, one in each trial) appeared in the center. The number spoken in Finnish was either "one," "two," "eight," or "nine" (one in each trial) and was presented through the computer speaker. The central letter ensured maintained eye fixation, and together with the spoken number, decreased the possibility of the participant of focusing only on the lateralized dot target(s). Although not relevant in this study, the central letter and the spoken number further offer the possibility to add additional conditions, in which the participant is required to also process these other stimuli in addition to the lateralized dot target(s).

In the present study, the participant was instructed to report only the position of the dot target(s) and to disregard the central letter and spoken number. Verbal guidance and a short training preceded the actual test session. The experimenter coded the participants' verbal responses to the computer (location of the dot target(s) in the "left," "right," or "both" side(s), or "no response"). Response time was not restricted. A new trial was initiated by the experimenter only after the participant had responded to the preceding trial. As the visual stimuli were only presented for $50 \mathrm{~ms}$, a screen with white noise followed the visual stimulus presentation and continued through the presentation of the spoken number until the experimenter coded the participant's response.
Correct responses and omissions for the dot target(s) were extracted and analyzed. A unilateral dot target was interpreted as "omitted" if the participant failed to correctly report the target's location (left or right). The bilateral dot targets' omissions were extracted as follows: If the participant incorrectly reported a dot target location as being "right" when it actually appeared bilaterally, the response was interpreted as "left omission" (cf. left-sided extinction); correspondingly, if the dot target was reported as "left" when it actually appeared bilaterally, the response was interpreted as "right omission" (cf. right-sided extinction).

\section{Assessment of non-lateralized visuoattentive deficits}

A novel application called the Active Space was used to generate a new assessment method, the Ball Rain task. The technical properties of the Active Space have previously been described in detail (Linnavuo, Kovalev, \& Sepponen, 2010; Rimminen, Lindström, Linnavuo, \& Sepponen, 2010; Villarreal et al., 2020). Briefly, a short throw video projector (Epson EB-680; Seiko EPSON Corporation, Suwa, Japan) was used to generate the visual stimuli. The projector produced a $173 \times 277 \mathrm{~cm}$ display on the wall. The pixel size was $1.9 \times 1.9 \mathrm{~mm}$. The participant was seated facing the screen at $180 \mathrm{~cm}$ distance with the midpoint located $120 \mathrm{~cm}$ from the floor. The visual angle was approximately $51^{\circ}$ vertically and $75^{\circ}$ horizontally. The control of the method and the task application were implemented using LabVIEWTM systems engineering software (National Instruments, Austin, Texas, USA).

The main technical parameters of the Ball Rain task are presented in Table 1. Each participant was instructed to observe colored spheres continuously appearing from the top of the screen in a fast downward motion, and they were instructed to react only to red spheres. The spheres could descend from any location along the superior border of the display, all the way out to the left and right extremes. There was no central fixation point, and eye movements were not monitored. The duration of the task was 3 minutes, and the actual test session was preceded by a short training, including verbal guidance. Correct reactions and reaction times, as well as missed targets, were extracted. For primary reaction time analyses, we used an approach utilized in several previous studies examining spatial attention (Anderson, Mennemeier, \& Chatterjee, 2000; Deouell et al., 2005; Villarreal et al., 2020). If the participant did not react to the target within $1000 \mathrm{~ms}$, the target was interpreted as "missed." If the participant responded earlier than $250 \mathrm{~ms}$ after the target onset, the reaction was excluded as anticipatory error. Reaction times that deviated more than 2.5 standard deviations from the mean were excluded as outliers. These exclusions were carried out separately for each participant and hemifield. A total of 3\% of all reactions were excluded in the left and right hemifields, respectively. A visualization of the Ball Rain task is presented in Figure 1. 
Table 1. Main technical parameters of the Ball Rain task

\begin{tabular}{ll}
\hline \hline Technical parameter & Ball Rain task description \\
\hline Task paradigm & $\begin{array}{c}\text { Colored non-overlapping spheres appear randomly from random horizontal locations on top of } \\
\text { the display and move downwards. Reaction to target with mouse button } 4 . \\
\text { Red sphere RGB: } 190,0,0\end{array}$ \\
Target & Until response, maximum $700 \mathrm{~ms}$ \\
Target display time & 50 on left, 50 on right side of display \\
Target locations & 250 to $1000 \mathrm{~ms}$ after target onset \\
Response window & Spheres other colored than red: Navy blue (RGB:0,0,100); Blue $(0,0,255) ;$ Cyan $(0,255,255) ;$ \\
Distraction stimuli & Yellow $(255,255,0) ;$ Dark green $(0,100,0) ;$ Pine Green $(0,100,100) ;$ Green $(0,255,0) ;$ Violet \\
& $(100,0,100) ;$ Pink $(255,0,255)$ \\
Distraction display time & $700 \mathrm{~ms}$ \\
Sphere diameter & $100 \mathrm{~mm}$ \\
Sphere velocity & $1.1 \mathrm{~m} / \mathrm{s}$ \\
Screen background & Solid Gray RGB:127,127,127 \\
ISI & Time between two stimulus onsets. Random, minimum 0 ms, average $175 \mathrm{ms,} \mathrm{maximum} 750 \mathrm{ms.}$ \\
ITI & Time between two target onsets. Random, minimum 1200 ms, average $1766 \mathrm{ms,} \mathrm{maximum}$ \\
Task duration & 4900 ms. \\
\hline \hline
\end{tabular}

Note: RGB, red, green, blue color model triplet; ISI, interstimulus interval; ITI, intertarget interval.

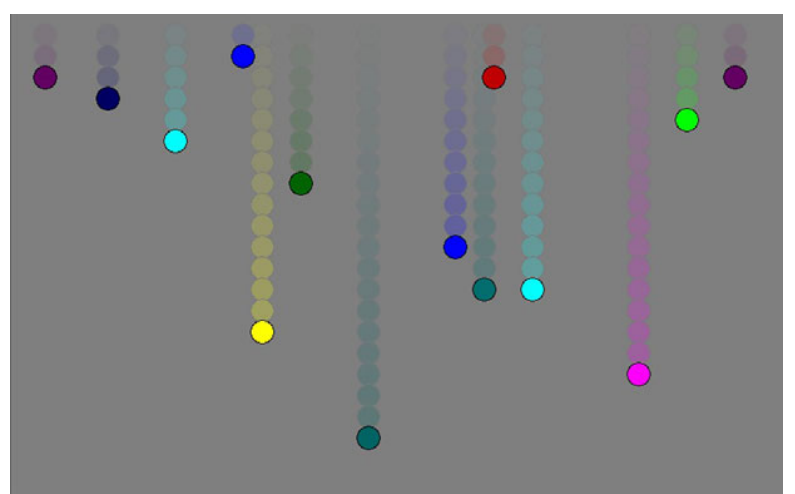

Fig. 1. Visualization of the Ball Rain task. Red target sphere appearing on the right side of display (for task parameters, see Table 1).

\section{Data Analyses}

Variables related to the assessment of contralesional omissions were created using a procedure described by Blini and co-workers (2016). First, an asymmetry index was calculated for each participant: omissions of the left hemifield were subtracted from those of the right hemifield. This way, negative values indicate the predominance of left-sided omissions (i.e., contralesional deficits in RH patients), and correspondingly, positive values indicate the predominance of right-sided omissions (i.e., contralesional deficits in LH patients). A value of zero indicates that omissions are distributed equally between the left and right hemifields, and thus, there is no spatial bias. Asymmetry indices were calculated separately for the unilateral and bilateral targets of the Lateralized Targets Computer Task and for the Bells Test. After calculating each participant's asymmetry index, the differences from the expected zero within the groups and the group differences were analyzed. Variables of the Ball Rain task were created by calculating omissions and average reaction times separately for the left and right hemifields.

The Statistical Package for the Social Sciences (IBM SPSS Statistics for Windows, Version 25.0. Armonk, New York: IBM Corporation) was used for statistical analyses. Reaction times were analyzed using a mixed analysis of variance (ANOVA) with Group (RH patients vs. LH patients vs. controls) as the between-subjects factor and Hemifield (left vs. right) as the within-subjects factor. For multiple pairwise comparisons, $p$ values were adjusted using the Bonferroni correction. All other variables were analyzed using nonparametric methods because of the skewness in distributions. Between-groups comparisons were analyzed using the Kruskal-Wallis test $\left(\chi^{2}\right)$. Dunn's test was used for post-hoc analyses. A step-down multiple hypotheses testing procedure (Benjamini \& Liu, 1999) was used to control the family-wise error. This procedure is a variation of the statistical approach pioneered by Benjamini and Hochberg (1995) to control the False Discovery Rate (FDR). Within-group analyses (the differences between the two hemifields) were performed using Wilcoxon signed-rank test. Effect sizes were calculated by computing partial eta squared $\left(\eta_{\text {partial }}^{2}\right)$ for mixed ANOVA, eta squared $\left(\eta^{2}\right)$ for the Kruskal-Wallis test, $r$ for Dunn's and Wilcoxon signed-rank tests and d for Bonferroni (Cohen, 1988; Tomczak \& Tomczak, 2014). For significant group differences, Cohen's descriptions for

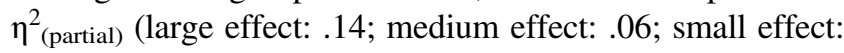
.01 ), $r$ (large effect: .5; medium effect: .3; small effect: .1), and d (large effect: .8; medium effect: .5; small effect: .2) were used (Cohen, 1988). The level of statistical significance was set at .05 . The $95 \%$ confidence intervals were reported where appropriate. 
Table 2. Average asymmetry indices and related within-group analyses of LH and RH patients and controls

\begin{tabular}{|c|c|c|c|c|}
\hline Within-group analyses: Deviation of asymmetry index from zero ${ }^{a}$ & $\underset{\text { index }^{\mathrm{b}}}{\text { Average asymmetry }}$ & Statistics (Z) & $p$ Value $^{\mathrm{c}}$ & Effect size $^{\mathrm{d}}$ \\
\hline \multicolumn{5}{|l|}{ Lateralized Targets Computer Task, bilateral targets } \\
\hline LH patients $(n=20)$ & .55 & 1.119 & .263 & \\
\hline RH patients $(n=20)$ & -.70 & -2.488 & .013 & $r=-.56^{* * *}$ \\
\hline Controls $(n=20)$ & .00 & .000 & 1.000 & \\
\hline \multicolumn{5}{|l|}{ Lateralized Targets Computer Task, unilateral targets } \\
\hline LH patients $(n=20)$ & .15 & .905 & .366 & \\
\hline RH patients $(n=20)$ & -.40 & -1.000 & .317 & \\
\hline Controls $(n=20)$ & .10 & .816 & .414 & \\
\hline \multicolumn{5}{|l|}{ The Bells Test } \\
\hline LH patients $(n=20)$ & .15 & 1.359 & .174 & \\
\hline RH patients $(n=20)$ & -.25 & .942 & .346 & \\
\hline Controls $(n=20)$ & -.45 & 1.425 & .154 & \\
\hline
\end{tabular}

LH, left hemisphere stroke; $\mathrm{RH}$, right hemisphere stroke.

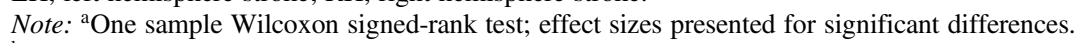

${ }^{b}$ Asymmetry index represents the spatial distribution of target omissions: negative values indicate predominance of left-sided omissions, positive values predominance of right-sided omissions, and zero expected equality in left-sided and right-sided omissions.

${ }^{c} p$ values smaller than .05 are statistically significant (marked in bold).

${ }^{\mathrm{d}}$ Effect sizes according to Cohen, 1988: $r=*$ small $>.1$, **medium $>.3$, ***large $>.5$.

Table 3. Group comparisons of the asymmetry indices of $\mathrm{LH}$ and $\mathrm{RH}$ patients and controls

\begin{tabular}{|c|c|c|c|c|c|c|c|c|c|c|c|}
\hline $\begin{array}{l}\text { Between-groups analyses: } \\
\text { Differences in asymmetry } \\
\text { indices }^{\mathrm{a}}\end{array}$ & & $\begin{array}{c}\mathrm{LH} \\
\text { patients, } \\
n=20\end{array}$ & $\begin{array}{c}\mathrm{RH} \\
\text { patients, } \\
n=20\end{array}$ & $\begin{array}{l}\text { Controls, } \\
n=20\end{array}$ & $\begin{array}{l}\text { Statistics } \\
\left(\chi^{2}\right)\end{array}$ & df & $\begin{array}{l}\text { Observed } \\
p \text { values }^{\mathrm{b}}\end{array}$ & $\begin{array}{l}\text { Adjusted } \\
p \text { values }^{\mathrm{c}}\end{array}$ & Rank & $\begin{array}{l}\text { Critical } \\
p \text { values }^{\mathrm{c}}\end{array}$ & $\begin{array}{c}\text { Effect } \\
\text { size }^{\mathrm{d}}\end{array}$ \\
\hline $\begin{array}{l}\text { Lateralized Targets Computer } \\
\text { Task, bilateral targets }\end{array}$ & & & & & 9.032 & 2 & .011 & & & & $\eta^{2}=.12 * *$ \\
\hline Post-hoc comparisons & $\begin{array}{l}\text { Mean } \\
\text { ranks }\end{array}$ & 35.02 & 22.65 & 33.83 & & & & & & & \\
\hline $\mathrm{RH}$ patients vs. LH patients & & & & & -12.375 & & .006 & .018 & 1 & .017 & $r=-.43 * *$ \\
\hline $\mathrm{RH}$ patients vs. controls & & & & & -11.175 & & .014 & .019 & 2 & .038 & $r=-.39 * *$ \\
\hline Controls vs. LH patients & & & & & 1.200 & & 1.000 & NS & 3 & .150 & \\
\hline $\begin{array}{l}\text { Lateralized Targets Computer } \\
\text { Task, unilateral targets }\end{array}$ & & & & & 1.683 & 2 & .431 & & & & \\
\hline The Bells Test & & & & & 3.625 & 2 & .163 & & & & \\
\hline
\end{tabular}

LH, left hemisphere stroke; RH, right hemisphere stroke; NS, not significant.

Note: ${ }^{a}$ Kruskall-Wallis test; post-hoc comparisons, mean ranks, and effect sizes presented for significant group differences.

${ }^{\mathrm{b}}$ Post-hoc comparisons: Observed $p$ values smaller than the critical $p$ values are statistically significant (marked in bold).

${ }^{\mathrm{c}}$ A step-down multiple hypotheses testing procedure; adjusted and critical $p$ values extracted to control the family-wise error.

${ }^{\mathrm{d}}$ Effect sizes according to Cohen, $1988: \eta^{2}=*^{*}$ small $>.01$, **medium $>.06, * * *$ large $>.14$ and $r=*$ small $>.1, * *$ medium $>.3, * * *$ large $>.5$.

\section{RESULTS}

\section{Asymmetry Indices of the Lateralized Targets Computer Task and the Bells Test}

Average asymmetry indices of the Lateralized Targets Computer Task and the Bells Test and related within-groups analyses are presented in Table 2. In the Lateralized Targets Computer Task, RH patients' asymmetry indices for bilateral targets were typically negative and differed significantly from the expected zero. This indicates the predominance of left-sided omissions (see Method section). The asymmetry indices for bilateral targets of the controls and LH patients did not differ significantly from zero. There were no significant deviations from zero in the asymmetry index for unilateral targets in any of the groups on either the Lateralized Targets Computer Task or the Bells Test.

Between-groups comparisons are presented in Table 3. RH patients' asymmetry indices for bilateral targets differed significantly from those of the controls and the LH patients. No significant differences occurred between the controls' and LH patients' asymmetry indices for bilateral targets. There were no significant differences between the participant groups' asymmetry indices for the unilateral targets nor for the Bells Test. 

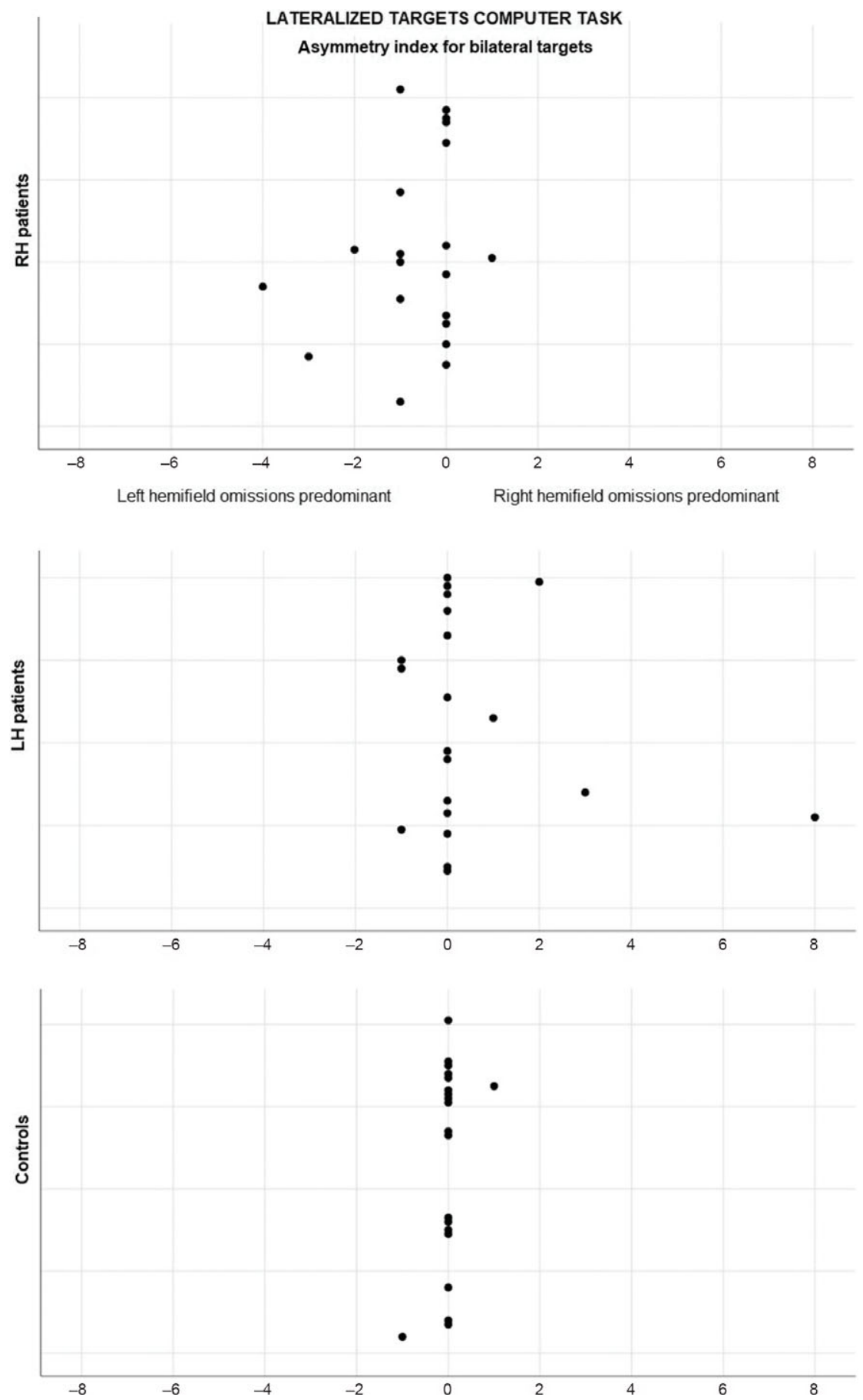

Fig. 2. Individual asymmetry indices for bilateral targets of the Lateralized Targets Computer Task.

In order to scrutinize each participant's asymmetry index individually, the indices for bilateral targets are presented in Figure 2.

\section{Response Omissions in the Ball Rain Task}

The average numbers of omissions in the Ball Rain task and related statistical comparisons between the groups and the two hemifields are reported in Table 4. RH and LH patients missed significantly more targets than the controls in both the left and right hemifields. No significant differences were observed between the patient groups. Neither RH or LH patients nor the controls showed omission differences between the two hemifields.

\section{Reaction Times in the Ball Rain Task}

The average reaction times of the participant groups and related statistical analyses are reported in Table 5. Both patient groups showed significantly slower reactions for 
Table 4. Average numbers of omissions in the Ball Rain task

\begin{tabular}{|c|c|c|c|c|c|c|c|c|c|c|c|}
\hline $\begin{array}{l}\text { Average omis- } \\
\text { sions }(\%) \text { and related } \\
\text { group comparisons }{ }^{\mathrm{a}}\end{array}$ & & $\begin{array}{c}\mathrm{LH} \\
\text { patients } \\
n=20\end{array}$ & $\begin{array}{c}\mathrm{RH} \\
\text { patients } \\
n=20\end{array}$ & $\begin{array}{c}\text { Controls } \\
n=20\end{array}$ & $\begin{array}{l}\text { Statistics } \\
\left(\chi^{2}\right)\end{array}$ & df & $\begin{array}{l}\text { Observed } \\
P \text { values }\end{array}$ & $\begin{array}{l}\text { Adjusted } \\
P \text { values }^{\mathrm{c}}\end{array}$ & Rank & $\begin{array}{l}\text { Critical } \\
P \text { values }^{\mathrm{c}}\end{array}$ & Effect size ${ }^{\mathrm{d}}$ \\
\hline $\begin{array}{l}\text { Ball Rain, left } \\
\text { hemifield }\end{array}$ & & $3 \%$ & $5 \%$ & $1 \%$ & 10.093 & 2 & .006 & & & & $\eta^{2}=.142 * * *$ \\
\hline $\begin{array}{l}\text { Post-hoc } \\
\text { comparisons }\end{array}$ & Mean ranks & 32.73 & 37.48 & 21.30 & & & & & & & \\
\hline $\begin{array}{l}\text { Controls vs. RH } \\
\text { patients }\end{array}$ & & & & & -16.175 & & .002 & .006 & 1 & .017 & $r=-.489 * *$ \\
\hline $\begin{array}{l}\text { Controls vs. LH } \\
\text { patients }\end{array}$ & & & & & -11.425 & & .029 & .038 & 2 & .038 & $r=-.345^{* *}$ \\
\hline $\begin{array}{c}\text { RH patients vs. } \\
\text { LH patients }\end{array}$ & & & & & 4.750 & & .364 & NS & 3 & .150 & \\
\hline $\begin{array}{l}\text { Ball Rain, right } \\
\text { hemifield }\end{array}$ & & $5 \%$ & $4 \%$ & $1 \%$ & 9.625 & 2 & .008 & & & & $\eta^{2}=.134 * *$ \\
\hline $\begin{array}{l}\text { Post-hoc } \\
\text { comparisons }\end{array}$ & Mean ranks & 33.80 & 36.58 & 21.12 & & & & & & & \\
\hline $\begin{array}{l}\text { Controls vs. RH } \\
\text { patients }\end{array}$ & & & & & -15.450 & & .004 & .012 & 1 & .017 & $r=-.460 * *$ \\
\hline $\begin{array}{l}\text { Controls vs. LH } \\
\text { patients }\end{array}$ & & & & & -12.675 & & .017 & .022 & 2 & .038 & $r=-.377^{* *}$ \\
\hline $\begin{array}{l}\text { RH patients vs. } \\
\text { LH patients }\end{array}$ & & & & & 2.775 & & .601 & NS & 3 & .150 & \\
\hline $\begin{array}{l}\text { Omission comparisons } \\
\text { two hemifields }\end{array}$ & between the & & & Statistics & (Z) & \multicolumn{6}{|c|}{$p$ value } \\
\hline \multicolumn{12}{|l|}{ Ball Rain } \\
\hline LH patients & & & \multicolumn{3}{|c|}{-1.235} & \multicolumn{6}{|c|}{.217} \\
\hline $\mathrm{RH}$ patients & & & \multicolumn{3}{|c|}{-.578} & \multicolumn{6}{|c|}{.563} \\
\hline Controls & & & \multicolumn{3}{|c|}{-.905} & \multicolumn{6}{|c|}{.366} \\
\hline
\end{tabular}

LH, left hemisphere stroke; RH, right hemisphere stroke; NS, not significant.

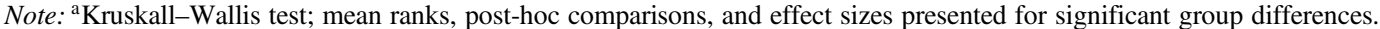

${ }^{b}$ Post-hoc comparisons: Observed $p$ values smaller than the critical $p$ values are statistically significant (marked in bold).

${ }^{\mathrm{c}}$ A step-down multiple hypotheses testing procedure; adjusted and critical $p$ values extracted to control the family-wise error.

${ }^{\mathrm{d}}$ Effect sizes according to Cohen, 1988: $\eta^{2}=*$ small $>.01$, **medium $>.06, * * * \operatorname{large}>.14$ and $r=*$ small $>.1, * *$ medium $>.3, * * *$ large $>.5$.

${ }^{\mathrm{e}}$ Paired Wilcoxon signed-rank test.

the Ball Rain targets than the controls did. The finding was equal in both hemifields since no significant effect of Hemifield or Hemifield $\times$ Group interaction was found.

\section{DISCUSSION}

The aim of the present study was to compare lateralized (i.e., contralesional) and non-lateralized (i.e., general) visuoattentive deficits in left and right hemisphere stroke patients. To assess the lateralized omissions, we used a traditional paper-and-pencil cancellation task, the Bells Test, and a Lateralized Targets Computer Task that is sensitive in detecting even subtle contralesional attention deficits. To assess the non-lateralized inattention, we developed the Ball Rain task, a new large-screen computer method in which we used moving visual stimuli and a setting requiring fastpaced selective attention. We examined whether these methodological qualities would make assessment sensitive in detecting non-lateralized inattention and whether the same patient groups also show contralesional attention deficits.
Our main findings are that both the $\mathrm{RH}$ and $\mathrm{LH}$ patients showed non-lateralized visual inattention and $\mathrm{RH}$ patients also showed subtle contralesional visuoattentive deficits. In the Ball Rain task, RH and LH patients missed significantly more targets than the controls did in both hemifields. Also, the reactions of the patient groups for the Ball Rain targets were significantly slower than those of the controls in both hemifields. In the Lateralized Targets Computer Task, RH patients missed significantly more left-sided than rightsided targets in bilateral trials and they missed significantly more left-sided targets in bilateral trials than the controls and LH patients. This spatial bias did not become evident with the Bells Test nor with the unilateral targets of the Lateralized Targets Computer Task. Hence, in the present study, assessment with a sensitive computer method was crucial in order to reveal RH patients' (subtle) contralesional attention deficits. LH patients showed no spatial bias in the Lateralized Targets Computer Task nor in the Bells Test, which ensures that the effect observed in RH patients is not a general byproduct of a brain lesion. 
Table 5. Average reaction times for the Ball Rain task of LH and RH patients and controls

\begin{tabular}{|c|c|c|c|c|c|c|c|}
\hline $\begin{array}{l}\text { Average reaction times, } \\
\text { ms (range) }\end{array}$ & $\begin{array}{l}\text { LH patients } \\
n=20\end{array}$ & $\begin{array}{c}\text { RH patients } \\
n=20\end{array}$ & $\begin{array}{c}\text { Controls } \\
n=20\end{array}$ & $\begin{array}{c}\text { Statistics } \\
\text { (Wilks } \lambda ; F)\end{array}$ & df & $P$ value $^{\mathrm{c}}$ & Effect size ${ }^{d}$ \\
\hline $\begin{array}{l}\text { Ball Rain, left } \\
\text { hemifield }\end{array}$ & $532(435-636)$ & $542(423-653)$ & $480(366-560)$ & & & & \\
\hline $\begin{array}{l}\text { Ball Rain, right } \\
\text { hemifield }\end{array}$ & $551(441-667)$ & $549(415-697)$ & $476(395-574)$ & & & & \\
\hline $\begin{array}{l}\text { Between-groups } \\
\text { comparisons }^{\mathrm{a}} \text { : }\end{array}$ & & & & 7.172 & 2 & .002 & $\eta_{\text {partial }}^{2}=.201 * * *$ \\
\hline \multirow[t]{2}{*}{$\begin{array}{l}\text { Post-hoc } \\
\text { comparisons }\end{array}$} & \multicolumn{3}{|c|}{$\begin{array}{c}95 \% \text { confidence interval } \\
\text { for difference }\end{array}$} & & & & \\
\hline & $\begin{array}{l}\text { Mean } \\
\text { difference }\end{array}$ & Lower bound & Upper bound & & & & \\
\hline $\begin{array}{l}\text { Controls vs. RH } \\
\text { patients }\end{array}$ & $-67.56 \mathrm{~ms}$ & $-116.98 \mathrm{~ms}$ & $-18.15 \mathrm{~ms}$ & & & .004 & $\mathrm{~d}=1.04 * * *$ \\
\hline $\begin{array}{l}\text { LH patients vs. RH } \\
\text { patients }\end{array}$ & $-3.89 \mathrm{~ms}$ & $-53.31 \mathrm{~ms}$ & $45.52 \mathrm{~ms}$ & & & 1.000 & \\
\hline $\begin{array}{l}\text { Controls vs. LH } \\
\text { patients }\end{array}$ & $-63.67 \mathrm{~ms}$ & $-113.09 \mathrm{~ms}$ & $-14.26 \mathrm{~ms}$ & & & .007 & $\mathrm{~d}=1.12^{* * *}$ \\
\hline \multicolumn{8}{|l|}{$\begin{array}{l}\text { Within-group } \\
\text { comparisons } \mathrm{b} \text { : }\end{array}$} \\
\hline Hemifield & & & & $.950 ; 2.986$ & 1 & .089 & \\
\hline Hemifield $\times$ Group & & & & $.914 ; 2.696$ & 2 & .076 & \\
\hline
\end{tabular}

LH, left hemisphere stroke; RH, right hemisphere stroke.

Note: Mixed ANOVA: ${ }^{\mathrm{B} B e t w e e n-g r o u p s}$ and

${ }^{\mathrm{b}}$ Within-group comparisons; effect sizes presented for significant group differences.

${ }^{c}$ For multiple pairwise comparisons, $p$ values adjusted by the Bonferroni correction; $p$ values smaller than .05 are statistically significant (marked in bold).

${ }^{\mathrm{d}}$ Effect sizes according to Cohen, 1988: $\eta_{\text {partial }}^{2}={ }^{*}$ small $>.01, * *$ medium $>.06, * * *$ large $>.14$ and $\mathrm{d}=*$ small $>.2, * *$ medium $>.5, * * *$ large $>.8$.

Supporting previous research (Battelli et al., 2001; Husain et al., 1997; Robertson et al., 1997), the results of the present study indicate that patients with unilateral brain damage may exhibit both non-lateralized and contralesional visuoattentive deficits. Significantly, this study complements the previous research (Chokron et al., 2019; Robertson et al., 1997; Robertson et al., 1998) by demonstrating that the connection between these two deficits is also observable in a clinical group whose contralesional attention deficits are only subtle. The observation is important both clinically and in terms of developing efficient assessment and rehabilitation methods. It stresses the importance of utilizing and developing different kinds of sensitive methods when assessing stroke patients' mild visuoattentive symptoms. As the Ball Rain task revealed non-lateralized attention deficits, the Lateralized Targets Computer Task was empirically confirmed as a purer measure of lateralized inattention. This might be due to a short presentation time in the latter one, which was likely to trigger contralesional omissions. The proposition is further supported by the fact that in our previous report (Villarreal et al., 2020), we also presented visual stimuli with short presentation time and found that the same RH patients showed significant contralesional but not ipsilesional omissions. The phenomenon became evident in two different computer tasks, but this time with unilateral targets. Therefore, it was actually confirmed that RH patients showed subtle neglect in addition to contralesional deficit under double stimulation evident in the present study.
It is essential to identify both lateralized and non-lateralized inattention since a) deficits in non-lateralized attention are a significant predictor of neglect (Robertson et al., 1997); b) non-lateralized attention deficits aggravate and prolong neglect (Hjaltason et al., 1996; Husain \& Rorden, 2003; Robertson, 2001; Samuelsson et al., 1998); c) along with recovery, a contralesional manifestation of the deficits shifts toward nonlateralized inattention (Nurmi et al., 2018); and d) neglect can be alleviated by improving non-lateralized attention (Robertson, Tegnér, Tham, Lo, \& Nimmo-Smith, 1995).

In the Ball Rain task, it is possible that the motion of the visual stimuli contributed to visuoattentive deficits being revealed as non-lateralized. Attention frequently has a crucial role in motion perception (Beer \& Röder, 2004; Cavanagh, 1992), and motion is important in attracting attention (Wolfe \& Horowitz, 2004). The phenomenon of shifting attention toward the side of motion has been demonstrated in healthy participants and in patients showing neglect (Hopfner et al., 2015). Moving cues in the contralesional hemispace alleviate neglect (Butter \& Kirsch, 1995; Butter, Kirsch, \& Reeves, 1990; Hopfner et al., 2015). Hence, at least in $\mathrm{RH}$ patients, one possible hypothesis might be that the motion of the Ball Rain stimuli acted as a "cue" that captured attention in the contralesional hemifield. As a result, their attention was distributed more evenly between the right and left visual fields. Nevertheless, their core attentional deficits presumably caused them to miss more targets than controls in both hemifields. 
One interesting point of view arises from the findings that attention is biased toward the ipsilesional space in patients with unilateral brain damage (Butler, Lawrence, Eskes, \& Klein, 2009; Gainotti, D'Erme, \& Bartolomeo, 1991; Mark, Kooistra, \& Heilman, 1988). Snow and Mattingley demonstrated (2006) that this ipsilesional preference, or "hyperattention," may lead to a deficit in inhibiting task-irrelevant information in the ipsilesional visual field (see also Bays, Singh-Curry, Gorgoraptis, Driver, \& Husain, 2010; Duncan, Humphreys, \& Ward, 1997; Shomstein, Lee, \& Behrmann, 2010). Therefore, it could be hypothesized that visuoattentive deficits in the ipsilesional hemifield might arise from different origins than those in the contralesional hemifield: the contralesional deficit might arise from dysfunctional spatial attention, and the ipsilesional deficit may be from a difficulty to filter and, thus, process information efficiently in the dynamic visual scene.

From a different perspective, it has been demonstrated that patients with chronic unilateral brain damage may show inappropriate saccades or bias to respond to contralesional stimuli (i.e. contralesional visual grasp) (Butter, Rapcsak, Watson, \& Heilman, 1988; Kwon \& Heilman, 1991). This phenomenon has been explained in accordance with the theory of DennyBrown and Chambers (1958): frontal lobes mediate avoidance behaviors (i.e. decreased allocation of attention) and the parietal lobes mediate approach behaviors (i.e. increased allocation of attention). In the healthy brain, these two regions inhibit the activity of each other. Therefore, unilateral damage to frontal areas may lead to disinhibition of parietal areas and, further, to strengthening of contralesional approach behavior (Butter et al., 1988; Kwon \& Heilman, 1991). As a consequence, allocation of attention increases in the contralesional hemispace and leads to ipsilesional attention deficit. In the present study, we did not use a central fixation point nor monitored eye movements in the Ball Rain task. Hence, it is possible that at least in some of the patients (e.g. the ones with anterior lesion), ipsilesional deficit is due to a contralesional visual grasp. The hypothesis is further supported by the fact (Kim et al., 1999) that this phenomenon may be taskdependent and may appear together with contralesional attention deficit (cf. RH patients showed contralesional and ipsilesional deficit in the Ball Rain task but only contralesional deficit in the Lateralized Targets Computer Task).

Certain caution is required when interpreting the results of the present study. First, these first-ever results with the new method should be confirmed with a larger sample size. Second, our test scenario in the Ball Rain task does not enable the differentiation of the factors (e.g., movement of the stimuli, high demands for visual selective attention, or both) to identify which caused the visuoattentive deficits of both of the patient groups to be exhibited as non-lateralized. Some studies have reported that patients with neglect show a normal perception of moving stimuli (Spinelli \& Zoccolotti, 1992) and deficits of selective attention only in the contralesional hemifield (Battelli et al., 2001). Future studies may bring additional information regarding the contributing factors in non-lateralized visuoattentive deficits of patients with subtle neglect and whether these deficits are associated with more severe symptomatology in other cognitive domains $(\mathrm{RH}$ and LH patients in this study did not differ from each other in other cognitive domains; see Villarreal et al., 2020). The possible effect of the fast-paced nature of the Ball Rain task on visuoattentive deficits should also be considered. The patients may have had limited capacity, which may have particularly affected the ipsilesional processing (as discussed above). This may have had an effect in bringing out both the contralesional and the ipsilesional attention deficits. Future studies may bring additional information on whether decelerating the stimulus presentation contributes in revealing "purer" contralesional attention deficits by suppressing the effect of limited processing capacity.

To conclude, we present a new method to assess visuoattentive deficits and demonstrate that computer-based assessment sensitively reveals various aspects of visual inattention. Patients with either right or left hemisphere stroke may suffer from non-lateralized visuoattentive deficits, and in right hemisphere stroke, these deficits may be accompanied by subtle contralesional inattention. Therefore, both kinds of deficits should be considered when assessing and rehabilitating these patients (Chokron et al., 2019; Husain \& Rorden, 2003; Robertson, 2001). New sensitive methods are needed in order to identify even minor attentional deficits. These deficits might not be detected with traditional assessment methods, but they might cause functional disability (Bonato \& Deouell, 2013; Bonato et al., 2012).

\section{ACKNOWLEDGMENTS}

This work was supported by Oskar Öflunds Stiftelse sr.; the University Hospital of Helsinki, Finland; and Alfred Kordelin Foundation. We thank Jari Lipsanen for statistical consultancy and Esko Ruuskanen for assistance in the raw data analysis, Viljami Salmela for consultations on visual perception, Kimmo Alho for commenting the manuscript, and Daniel Villarreal for assistance in proofreading the manuscript, our colleagues who assisted in the recruitment of their patients to the study, and the participants who generously gave their time to take part in this research.

\section{CONFLICT OF INTEREST}

The authors have no conflicts of interest to disclose.

\section{REFERENCES}

Anderson, B., Mennemeier, M., \& Chatterjee, A. (2000). Variability not ability: another basis for performance decrements in neglect. Neuropsychologia, 38, 785-796. doi: 10.1016/s0028-3932(99) 00137-2

Bartolomeo, P. \& Chokron, S. (2002). Orienting of attention in unilateral neglect. Neuroscience and Biobehavioral Reviews, 26, 217-234. doi: 10.1016/s0149-7634(01)00065-3

Battelli, L., Cavanagh, P., Intriligator, J., Tramo, M.J., Hénaff, M.A., Michèl, F., \& Barton, J.J. (2001). Unilateral right parietal 
damage leads to bilateral deficit for high-level motion. Neuron, 32, 985-995. doi: 10.1016/s0896-6273(01)00536-0

Bays, P.M., Singh-Curry, V., Gorgoraptis, N., Driver, J., \& Husain, M. (2010). Integration of goal- and stimulus-related visual signals revealed by damage to human parietal cortex. The Journal of Neuroscience, 30, 5968-5978. doi: 10.1523/JNEUROSCI. 0997-10.2010

Beer, A.L. \& Röder, B. (2004). Unimodal and crossmodal effects of endogenous attention to visual and auditory motion. Cognitive, Affective and Behavioral Neuroscience, 4, 230-240. doi: 10. 3758/cabn.4.2.230

Benjamini, Y. \& Hochberg, Y. (1995). Controlling the false discovery rate: a practical and powerful approach to multiple testing. Journal of the Royal Statistical Society: Series B (Methodological), 57, 289-300. doi: 10.1111/j.2517-6161.1995.tb02031.x

Benjamini, Y. \& Liu, W. (1999). A step-down multiple hypotheses testing procedure that controls the false discovery rate under independence. Journal of Statistical Planning and Inference, 82, 163-170. doi: 10.1016/S0378-3758(99)00040-3

Blini, E., Romeo, Z., Spironelli, C., Pitteri, M., Meneghello, F., Bonato, M., \& Zorzi, M. (2016). Multi-Tasking uncovers right spatial neglect and extinction in chronic left-hemisphere stroke patients. Neuropsychologia, 92, 147-157. doi: 10.1016/j. neuropsychologia.2016.02.028

Bonato, M. (2015). Unveiling residual, spontaneous recovery from subtle hemispatial neglect three years after stroke. Frontiers in Human Neuroscience, 9, 413. doi: 10.3389/fnhum. 2015.00413

Bonato, M. \& Deouell, L.Y. (2013). Hemispatial neglect: computerbased testing allows more sensitive quantification of attentional disorders and recovery and might lead to better evaluation of rehabilitation. Frontiers in Human Neuroscience, 7, 162. doi: 10.3389/fnhum.2013.00162

Bonato, M., Priftis, K., Marenzi, R., Umiltà, C., \& Zorzi, M. (2012). Deficits of contralesional awareness: a case study on what paperand-pencil tests neglect. Journal of Neuropsychology, 26, 20-36. doi: 10.1037/a0025306

Bonato, M., Romeo, Z., Blini, E., Pitteri, M., Durgoni, E., Passarini, L., ... Zorzi, M. (2019). Ipsilesional impairments of visual awareness after right-hemispheric stroke. Frontiers in Psychology, 10, 697. doi: 10.3389/fpsyg.2019.00697

Butler, B.C., Lawrence, M., Eskes, G.A., \& Klein, R. (2009). Visual search patterns in neglect: comparisons of peripersonal and extrapersonal space. Neuropsychologia, 47, 869-878. doi: 10. 1016/j.neuropsychologia.2008.12.020

Butter, C.M. \& Kirsch, N. (1995). Effect of lateralized kinetic visual cues on visual search in patients with unilateral spatial neglect. Journal of Clinical and Experimental Neuropsychology, 17, 856-867. doi: 10.1080/01688639508402435

Butter, C.M., Kirsch, N.L., \& Reeves, G. (1990). The effect of lateralized dynamic stimuli on unilateral spatial neglect following right-hemisphere lesions. Restorative Neurology and Neuroscience, 2, 39-46. doi: 10.3233/RNN-1990-2105

Butter, C.M., Rapcsak, S., Watson, R.T., \& Heilman, K.M. (1988). Changes in sensory inattention, directional motor neglect and "release" of the fixation reflex following a unilateral frontal lesion: a case report. Neuropsychologia, 26, 533-545. doi: 10. 1016/0028-3932(88)90110-8

Buxbaum, L., Ferraro, M., Veramonti, T., Farne, A., Whyte, J., La davas, E., ... Coslett, H. (2004). Hemispatial neglect: subtypes, neuroanatomy and disability. Neurology, 62, 749-756. doi: 10.1212/01.wnl.0000113730.73031.f4
Cavanagh, P. (1992). Attention-Based motion perception. Science, 257, 1563-1565. doi: 10.1126/science.1523411

Chokron, S., Peyrin, C., \& Perez, C. (2019). Ipsilesional deficit of selective attention in left homonymous hemianopia and left unilateral spatial neglect. Neuropsychologia, 128, 305-314. doi: 10.1016/j.neuropsychologia.2018.03.013

Cohen, J. (1988). Statistical Power Analysis for the Behavioral Sciences (2nd ed., pp. 25-27, 79-80, 283-287, 366-368). New York, NY: Lawrence Erlbaum Associates.

Denny-Brown, D. \& Chambers, R.A. (1958). The parietal lobe and behavior. Association for Research in Nervous and Mental Diseases Proceedings, 36, 35-117.

Deouell, L.Y., Sacher, Y., \& Soroker, N. (2005). Assessment of spatial attention after brain damage with a dynamic reaction time test. Journal of the International Neuropsychological Society, 11, 697-707. doi: 10.1017/S1355617705050824

Duncan, J., Humphreys, G., \& Ward, R. (1997). Competitive brain activity in visual attention. Current Opinion in Neurobiology, 7, 255-261. doi: 10.1016/s0959-4388(97)80014-1

Gainotti, G., D’Erme, P., \& Bartolomeo, P. (1991). Early orientation of attention toward the half space ipsilateral to the lesion in patients with unilateral brain damage. Journal of Neurology, Neurosurgery, and Psychiatry, 54, 1082-1089. doi: 10.1136/jnnp.54.12.1082

Gauthier, L., Dehaut, F., \& Joanette, Y. (1989). The Bells Test: a quantitative and qualitative test for visual neglect. International Journal of Clinical Neuropsychology, 11, 49-54.

George, M.S., Mercer, J.S., Walker, R., \& Manly, T. (2008). A demonstration of endogenous modulation of unilateral spatial neglect: the impact of apparent time-pressure on spatial bias. Journal of the International Neuropsychological Society, 14, 33-41. doi: 10. 1017/S135561770808003X

Halligan, P., Cockburn, J., \& Wilson, B. (1991). The behavioural assessment of visual neglect. Neuropsychological Rehabilitation, 1, 5-32. doi: 10.1080/09602019108401377

Heilman, K.M., Valenstein, E., \& Watson, R.T. (2000). Neglect and related disorders. Seminars in Neurology, 20, 463-470. doi: $10.1055 / \mathrm{s}-2000-13179$

Hjaltason, H., Tegnér, R., Tham, K., Levander, M., \& Ericson, K. (1996). Sustained attention and awareness of disability in chronic neglect. Neuropsychologia, 34, 1229-1233. doi: 10.1016/00283932(96)00044-9

Hopfner, S., Kesselring, S., Cazzoli, D., Gutbrod, K., Laube-Rosenpflanzer, A., Chechlacz, M., ... Nyffeler, T. (2015). Neglect and motion stimuli - insights from a touchscreen-based cancellation task. PLoS One, 10, e0132025. doi: 10.1371/journal. pone. 0132025

Husain, M. \& Rorden, C. (2003). Non-Spatially lateralized mechanisms in hemispatial neglect. Neuroscience, 4, 26-36. doi: $10.1038 / \mathrm{nrn} 1005$

Husain, M., Shapiro, K., Martin, J., \& Kennard, C. (1997). Abnormal temporal dynamics of visual attention in spatial neglect patients. Nature, 385, 154-156. doi: 10.1038/ $385154 \mathrm{a} 0$

Kim, D.Y., Ku, J., Chang, W.H., Park, T.H., Lim, J.Y., Han, K., . . . Kim, S.I. (2010). Assessment of post-stroke extrapersonal neglect using a three-dimensional immersive virtual street crossing program. Acta Neurologica Scandinavica, 121, 171-177. doi: 10.1111/j.1600-0404.2009.01194.x

Kim, M., Na, D.L., Kim, G.M., Adair, J.C., Lee, K.H., \& Heilman, K.M. (1999). Ipsilesional neglect: behavioural and anatomical features. Journal of Neurology, Neurosurgery and Psychiatry, 67, 35-38. doi: 10.1136/jnnp.67.1.35 
Kwon, S.E. \& Heilman, K.M. (1991). Ipsilateral neglect in a patient following a unilateral frontal lesion. Neurology, 41, 2001-2004. doi: 10.1212/wnl.41.12.2001

Linnavuo, M., Kovalev, O., \& Sepponen, R. (2010). Proactive space - a novel post stroke mobility rehabilitation tool. 2nd Circuits and Systems for Medical and Environmental Applications, Merida, Mexico.

Malhotra, P.A., Parton, A.D., Greenwood, R., \& Husain, M. (2006). Noradrenergic modulation of space exploration in visual neglect. Annals of Neurology, 59, 186-190. doi: 10.1002/ana.20701

Mark, V.W., Kooistra, C.A., \& Heilman, K.M. (1988). Hemispatial neglect affected by non-neglected stimuli. Neurology, 38, 1207-1211. doi: 10.1212/wnl.38.8.1207

Mukand, J.A., Guilmette, T.J., Allen, D.G., Brown, L.K., Brown, S.L., Tober, K.L., \& Vandyck, W.R. (2001). Dopaminergic therapy with carbidopa L-dopa for left neglect after stroke: a case series. Archives of Physical Medicine and Rehabilitation, 82, 1279-1282. doi: 10.1053/apmr.2001.25149

Nurmi, L., Ruuskanen, E.I., Nurmi, M., Koivisto, A.M., Parkkila, A.K., Numminen, H., ... Jehkonen, M. (2018). Occurrence and recovery of different neglect-related symptoms in right hemisphere infarct patients during a 1-year follow-up. Journal of the International Neuropsychological Society, 24, 617-628. doi: 10.1017/S1355617718000176.

Ogourtsova, T., Silva, W., Archambault, P., \& Lamontagne, A. (2017). Virtual reality treatment and assessment for post-stroke unilateral spatial neglect: a systematic literature review. Neuropsychological Rehabilitation, 27, 409-454. doi: 10.1080/09602 011.2015.1113187

Peskine, A., Box, N., Galland, A., Caron, E., Rautureau, G., Jouvent, R., \& Pradat-Diehl, P. (2011). Virtual reality assessment for visuospatial neglect: importance of a dynamic task. Journal of Neurology, Neurosurgery and Psychiatry, 82, 1407-1409. doi: 10.1136/jnnp.2010.217513

Rimminen, H., Lindström, J., Linnavuo, M., \& Sepponen, R. (2010). Detection of falls among the elderly by a floor sensor using the electric near field. IEEE Transactions on Information Technology in Biomedicine, 14, 1475-1476. doi: 10.1109/ TITB.2010.2051956

Robertson, I.H. (2001). Do we need the "lateral" in unilateral neglect? Spatially nonselective attention deficits in unilateral neglect and their implications for rehabilitation. NeuroImage, 14, 85-90. doi: 10.1006/nimg.2001.0838

Robertson, I.H., Halligan, P.W., Bergego, C., Hömberg, V., Pizzamiglio, L., Weber, E., \& Wilson, B.A. (1994). Right neglect following right hemisphere damage? Cortex, 30, 199-213. doi: 10.1016/s0010-9452(13)80193-1

Robertson, I.H., Manly, T., Beschin, N., Daini, R., Haeske-Dewick, H., Hömberg, V., .. We Weber, E. (1997). Auditory sustained attention is a marker of unilateral spatial neglect. Neuropsychologia, 35, 1527-1532. doi: 10.1016/s0028-3932(97)00084-5

Robertson, I.H., Mattingley, J.B., Rorden, C., \& Driver, J. (1998). Phasic alerting of neglect patients overcomes their spatial deficit in visual awareness. Nature, 395, 169-172. doi: 10.1038/25993

Robertson, I.H., Tegnér, R., Tham, K., Lo, A., \& Nimmo-Smith, I. (1995). Sustained attention training for unilateral neglect: theoretical and rehabilitation implications. Journal of Clinical and Experimental Neuropsychology, 17, 416-430. doi: 10.1080/ 01688639508405133

Rueckert, L., \& Grafman, J. (1996). Sustained attention deficits in patients with right frontal lesions. Neuropsychologia, 34, 953-963. doi: 10.1016/0028-3932(96)00016-4

Rueckert, L. \& Grafman, J. (1998). Sustained attention deficits in patients with lesions of posterior cortex. Neuropsychologia, 36, 653-660. doi: 10.1016/s0028-3932(97)00150-4

Samuelsson, H., Hjelmquist, E.K., Jensen, C., Ekholm, S., \& Blomstrand, C. (1998). Nonlateralized attentional deficits: an important component behind persisting visuospatial neglect? Journal of Clinical and Experimental Neuropsychology, 20, 73-88. doi: 10.1076/jcen.20.1.73.1481

Shomstein, S., Lee, J., \& Behrmann, M. (2010). Top-Down and bottom-up attentional guidance: investigating the role of the dorsal and ventral parietal cortices. Experimental Brain Research, 206, 197-208. doi: 10.1007/s00221-010-2326-Z

Snow, J.C. \& Mattingley, J.B. (2006). Goal-Driven selective attention in patients with right hemisphere lesions: how intact is the ipsilesional field? Brain, 129, 168-181. doi: 10.1093/brain/ awh690

Spinelli, D. \& Zoccolotti, P. (1992). Perception of moving and stationary gratings in brain damaged patients with unilateral spatial neglect. Neuropsychologia, 30, 393-401. doi: 10.1016/00283932(92)90112-y

Tomczak, M. \& Tomczak, E. (2014). The need to report effect size estimates revisited. An overview of some recommended measures of effect size. Trends in Sport Science, 1, 19-25.

van Kessel, M.E., van Nes, I.J., Brouwer, W.H., Geurts, A.C., \& Fasotti, L. (2010). Visuospatial asymmetry and non-spatial attention in subacute stroke patients with and without neglect. Cortex, 46, 602-612. doi: 10.1016/j.cortex.2009.06.004

Villarreal, S., Linnavuo, M., Sepponen, R., Vuori, O., Jokinen, H., \& Hietanen, M. (2020). Dual-Task in Large Perceptual Space Reveals Subclinical Hemispatial Neglect. Journal of the International Neuropsychological Society, 1-13. doi: 10.1017/ S1355617720000508

Wilkins, A.J., Shallice, T., \& McCarthy, R. (1987). Frontal lesions and sustained attention. Neuropsychologia, 25, 359-365. doi: 10. 1016/0028-3932(87)90024-8

Williamson, J.B., Lamb, D.G., Burtis, D.B., Haque, S., Zilli, E.M., Kesayan, T., ... Heilman, K.M. (2018). Right hemispatial ipsilesional neglect with chronic right hemisphere strokes. Journal of Clinical and Experimental Neuropsychology, 40, 347-356. doi: 10.1080/13803395.2017.1347606

Wolfe, J.M. \& Horowitz, T.S. (2004). What attributes guide the deployment of visual attention and how do they do it? Nature Reviews Neuroscience, 5, 495-501. doi: 10.1038/nrn1411 